Check for updates

Cite this: RSC Adv., 2017, 7, 30663

Received 25th March 2017 Accepted 29th May 2017

DOI: $10.1039 / \mathrm{c} 7 \mathrm{ra03466k}$

rsc.li/rsc-advances

\section{Process development, friction insensitivity improvement and compatibility studies of tetranitroacetimidic acid $\uparrow$}

\author{
Pandurang M. Jadhav, (D) *a Hima Prasanth, ${ }^{\text {a }}$ Raj K. Pandey, ${ }^{a}$ Rajendra S. Patil ${ }^{b}$ \\ and Sibes C. Bhattacharyya ${ }^{\mathrm{b}}$
}

Herein, nitration of 1,1-diamino-2,2-dinitroethene (FOX-7) using concentrated nitric acid was studied in detail to establish a process for the preparation of tetranitroacetimidic acid (TNAA), an environmentally benign oxidizer. Nitration has also been studied in the presence of various solvents that facilitate liquidliquid reactions. The products have been characterized by thermal and spectroscopic techniques. The process was kinetically evaluated, and a second order rate with respect to the concentration of FOX-7 was observed. Friction insensitivity of TNAA improved to $196 \mathrm{~N}$ by altering the particle size and shape after precipitation in various alkanes. The improvement of the TNAA crystal shape was observed via a scanning electron microscope (SEM). Brunauer-Emmett-Teller (BET) analysis indicates sub-micron particle size and large surface area of TNAA. Compatibility of TNAA with composite propellant ingredients, mainly toluene diisocyanate (TDI) and hydroxyl-terminated polybutadiene (HTPB), was assessed by monitoring the Fourier transform infrared spectroscopy (FTIR) spectra. Thermogravimetric analysis (TGA) of TNAA with ammonium perchlorate was also studied to observe the decomposition pattern in view of partial replacement of ammonium perchlorate in propellant formulations. Overall, this study has revealed the process development outcomes, methodology of improving the friction insensitivity of TNAA, and applicability of TNAA in composite propellant formulations.

\section{Introduction}

For several decades, ammonium perchlorate (AP) has exclusively been utilized as an oxidizer in propellants of space and military rocket motors mainly due to its excellent oxygen balance and density. Moreover, ammonium perchlorate-based composite propellants (APCPs), containing a typical composition of aluminum and polybutadiene binders, provide a large magnitude of specific impulse. ${ }^{1}$ Applications of APCPs have also gained importance in pyrotechnic devices (warning flares and fireworks), air bag inflators, and aircraft ejection seats due to their simplicity and reliability. ${ }^{2}$ On the other hand, AP has been detected as toxic to the environment to a large extent. Owing to its high stability, solubility in water, and its multiple applications, it remains widely distributed in surface and groundwater systems world-wide and particularly in the south-western US $^{3}$. In vertebrates, perchlorate affects the growth and development because thyroids bind with it instead of binding with iodide. The pigmentation and growth of amphibians is also disturbed due to exposure to AP. ${ }^{4}$ In addition,

${ }^{a}$ High Energy Materials Research Laboratory, Pune, India-411021. E-mail: jadhav. pm@hemrl.drdo.in

${ }^{b}$ Advanced Centre for Energetic Materials, Nasik, India-422009

$\dagger$ Electronic supplementary information (ESI) available. See DOI: $10.1039 / \mathrm{c} 7 \mathrm{ra} 03466 \mathrm{k}$ exhaust gases of APCPs are rich in perchlorate and hydrogen chloride $(0.45$ to $1 \mathrm{~mol} / 100 \mathrm{~g}$ of propellant), which deplete the ozone layer and can also cause acid rain. ${ }^{5}$ Due to this, worldwide importance has been given to the development of chlorine-free oxidizers with equivalent or better performance.

The cheapest and easily available oxidizer ammonium nitrate (AN) was considered as a substitute to AP in the formulation of a composite propellant. However, its usage in large rocket motors has shown limitations due to its adverse characteristics such as hygroscopicity, near room temperature phase transformation involving a volume change, and low burning rate (BR) and energetics. ${ }^{6}$ Ammonium dinitramide (ADN), a salt of ammonia cation $\left(\mathrm{NH}_{4}^{+}\right)$and dinitramide anion $\left(\mathrm{N}^{+}\left(\mathrm{NO}_{2}\right)_{2}{ }^{-}\right)$, contains $26 \%$ oxygen balance and is materialized as a chlorine-free oxidizer for composite propellant formulations. However, its processability, economy, thermal stability, and hygroscopicity are major concerns. ${ }^{7}$ Propellants containing hydrazinium nitroformate (HNF), oxygen rich species (13\%), have shown rapid burning with high combustion efficiency. However, the thermal stability, friction sensitivity, and hydrazinium cation of HNF are severe issues in view of its applications. ${ }^{8}$ Recently, tetranitroacetimidic acid $\left(\mathrm{C}_{2} \mathrm{HN}_{5} \mathrm{O}_{9}\right)$ has emerged as a potential chlorine-free oxidizer possessing higher oxygen balance. Several properties of TNAA have been found to be comparable with those of AP, ADN, and HNF. ${ }^{9}$ 
However, its low friction sensitivity has limited its application in composite propellant formulations.

Hence, the present study was aimed at the development of tetranitroacetimidic acid (TNAA) with improved characteristics, and its compatibility with other composite propellant ingredients was also studied.

\section{Experimental procedures}

Information on the synthesis of TNAA from the nitration of 1,1diamino-2,2-dinitroethene (FOX-7) was found to be scanty in terms of process development activities. Hence, process developmental studies of TNAA in detail were planned to obtain the operating envelope, reaction kinetics, and process know-how for scale up. For verifying the applicability of TNAA in propellant formulations, experiments were designed for the improvement of its friction insensitivity and monitoring its reactivity with other ingredients of the composite propellant. The details of the experiments for these objectives are provided in the following subsections.

\subsection{Synthesis and characterization}

Nitration of 1,1-diamino-2,2-dinitroethene (FOX-7) was carried out under ambient condition using excess fuming nitric acid in various proportions ranging from 14 to 50 per mole of FOX-7. The reaction scheme (Fig. $\mathrm{S} 1 \dagger$ ) and characterization details of TNAA are given in the ESI. $\uparrow$ TNAA was obtained as a white solid on evacuating excess nitric acid and crystallization from dichloromethane. The reaction was also carried out at 15,25 , and $35^{\circ} \mathrm{C}$ to observe the reaction pace. The product yield was obtained in the range of 85$95 \%$. The process flow diagram for the TNAA synthesis is shown in Fig. 1.

Furthermore, solvents such as dichloromethane (DCM), carbon tetrachloride, dimethylformamide (DMF), formamide, etc. were introduced in the reaction for improving the processability. The products obtained during the process development studies were characterized by spectroscopic, thermal, and chromatographic techniques. Elemental analysis was carried out using a VarioMICRO instrument of Elementar Analysensysteme GmbH. The IR

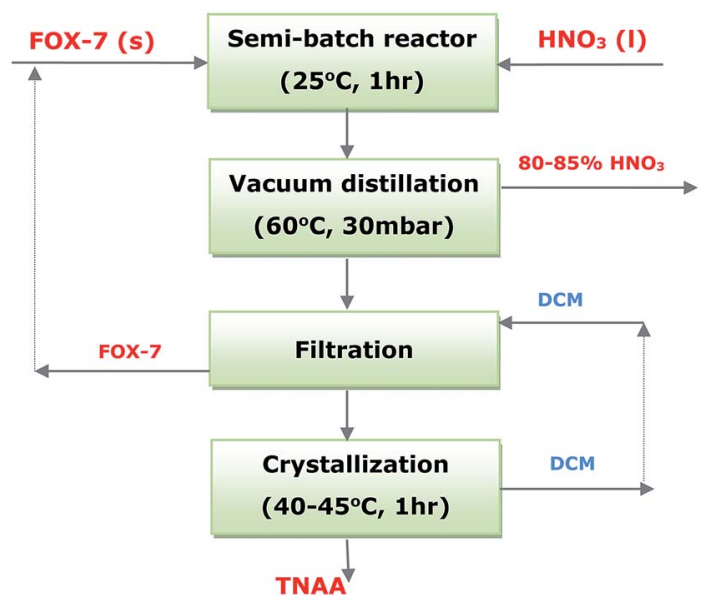

Fig. 1 Process flow diagram for the TNAA synthesis. spectra were obtained using a Thermofisher FTIR (model-Nicolate iS50 FTIR) spectrophotometer in the ATR mode. ${ }^{1} \mathrm{H}$ and ${ }^{13} \mathrm{C}$ nuclear magnetic resonance (NMR) spectra of the solid samples were obtained using a Bruker MSL-300. Differential scanning calorimetry was carried out using a PerkinElmer DSC-7 instrument at the heating rate of $10{ }^{\circ} \mathrm{C} \mathrm{min}^{-1}$ in a nitrogen atmosphere, and thermogravimetric analysis was carried out using a thermal analyzer of the TA instrument SDTA Q600.

\subsection{Friction insensitivity improvement}

A $130 \mathrm{~g} \mathrm{~L}^{-1}$ solution of TNAA in DCM was prepared at $25{ }^{\circ} \mathrm{C}$ and precipitated in pentane, hexane, cyclohexane, and heptane under ambient conditions and constant stirring. Solution of TNAA to anti-solvent ratio was fixed at $1: 4$ by volume to ensure maximum possible yield of precipitation. Morphology of the precipitated samples was analyzed using scanning electron microscope (SEM) of Philips Company for investigating the particle shape and size. Impact and friction sensitivity of the precipitated samples were also measured by fall hammer and Julius Peters apparatus method, respectively. Further surface area and particle size for the samples were analyzed using a BET instrument of Micromeritics Corporation.

\subsection{Compatibility studies with propellant ingredients}

The reactivity of TNAA with HTPB and TDI was separately monitored as well as in mixed form through spectroscopy. Fourier transform infrared spectra (FTIR) were obtained at frequent intervals using a Nicolet iS10 spectrometer of Thermo Scientific. Analyses were carried out under attenuated transmission mode (ATR) under the conditions of spectral range of $4000-500 \mathrm{~cm}^{-1}, 32$ scans, and $4 \mathrm{~cm}^{-1}$ resolution. Furthermore, thermal analysis of TNAA with ammonium perchlorate to view partial replacement was carried out using SDTA Q600Thermal analyzer of TA Instruments. A mixture of an equivalent quantity of TNAA and AP was prepared, and the decomposition patterns were obtained for a $0.2 \mathrm{mg}$ sample at a $5{ }^{\circ} \mathrm{C}$ per minute heating rate. Commercial grade ingredients were used for the compatibility study (HTPB: M/s Anabond Pvt. Ltd., TDI (80:20):M/s Bayer Chemicals, AP:M/s Pandian Chemicals Pvt. Ltd.).

\section{Results and discussion}

In the current scenario, there is a dire need of chlorine free as well as stabilized oxidizers for propellant formulations in the civil and defense sectors. Usually, a large content of oxidizer is preferred in propellant formulations for obtaining optimal performance characteristics. This requirement of oxidizer can only be met by carrying out the process development studies to realize the suitable method for the scale-up of the process. Hence, the present study was targeted at obtaining complete insights into the process development and kinetics of TNAA synthesis.

\subsection{Process development studies}

The desired quantity of nitric acid is always essential for obtaining higher yields along with minimal effluent content. This also facilitates an increasing throughput for a given 


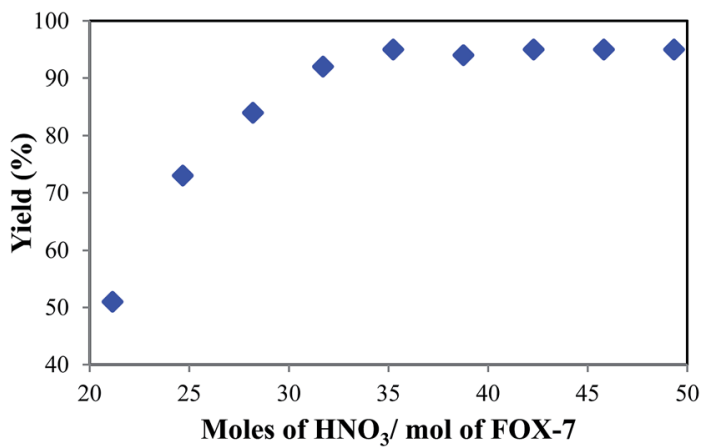

Fig. 2 Effect of nitric acid quantity on the TNAA yield.

volume of reactor. The studies for determining the optimal quantity of nitric acid were carried out considering four moles of nitric acid per mole of FOX-7. This is because of the noted requirement of two nitronium ions per mole of TNAA, which are likely obtained upon the dissociation of four moles of nitric acid. ${ }^{10}$ However, TNAA was not obtained at four or five moles of nitric acid. This was due to incomplete dissociation of nitric

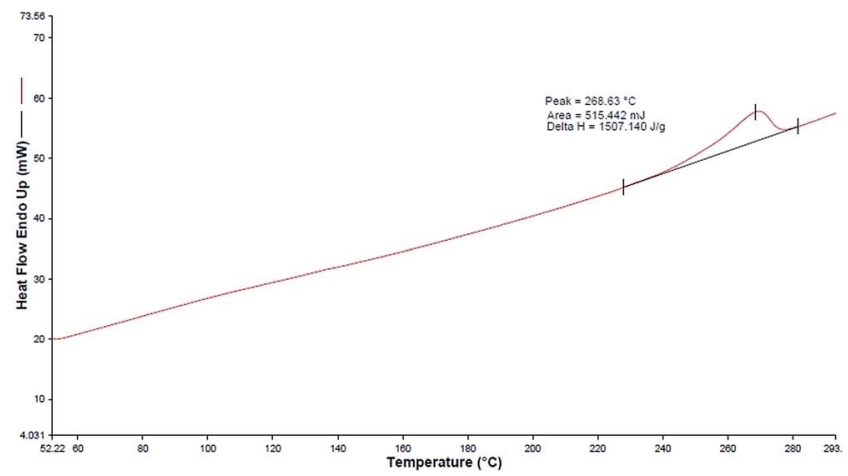

(a)

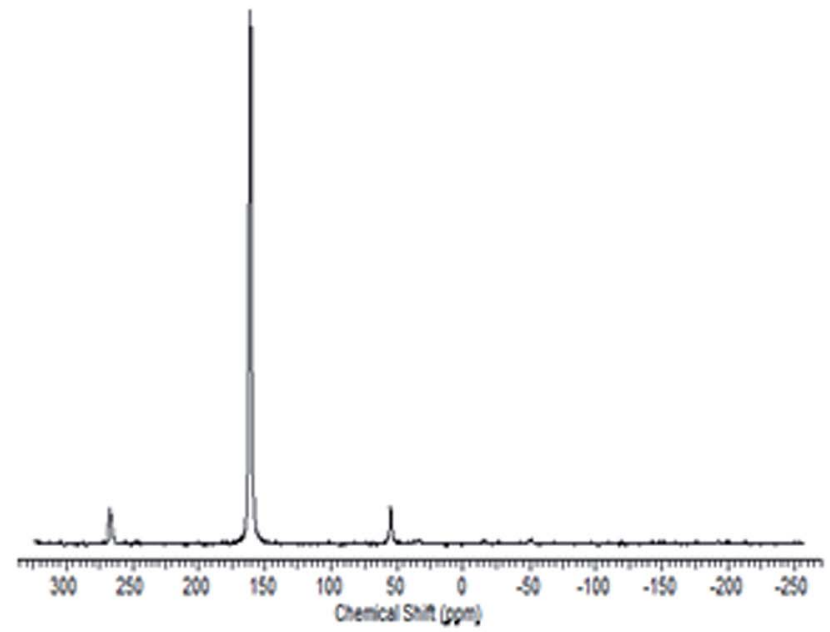

(c) acid to nitronium ion and also generation of a water molecule in the reaction, which hydrolyzed TNAA. On further increasing the number of moles of nitric acid, TNAA was obtained. The observed yield of TNAA against nitric acid mole/FOX-7 is plotted in Fig. 2.

The steep increase in yield was obtained on increasing the nitric acid quantity from 21 to $32 \mathrm{~mol} \mathrm{~mol}^{-1}$ of FOX-7. In this region, hydrolysis of TNAA was averted due to the presence of excess nitric acid. The maximum yield of TNAA was found to be $95 \%$ at 35 mole of nitric acid/mole of FOX-7. Further increase in the nitric acid quantity did not facilitate an improvement in the TNAA yield. Moreover, this region required additional efforts for removing nitric acid during distillation.

Furthermore, a liquid-liquid reaction was attempted to establish a method for continuous processing. The solubility of FOX-7 was found to be significant in DMSO (dimethyl sulfoxide), DMF ( $N, N$-dimethylformamide), and NMP ( $N$-methyl-2pyrrolidone). ${ }^{11}$ DMSO and NMP were not found to be suitable because decomposition of TNAA was noticed in them. Similar observations were also reported during NMR analysis of TNAA. ${ }^{9}$

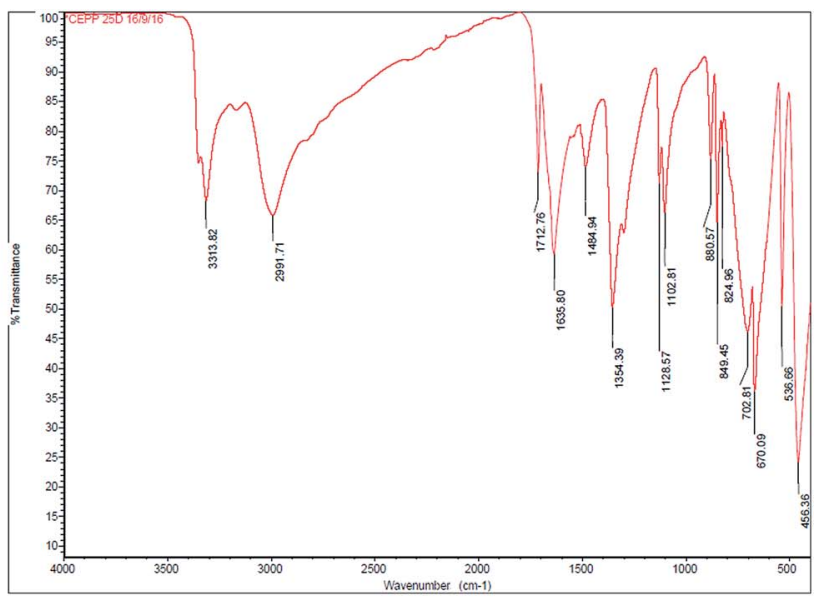

(b)

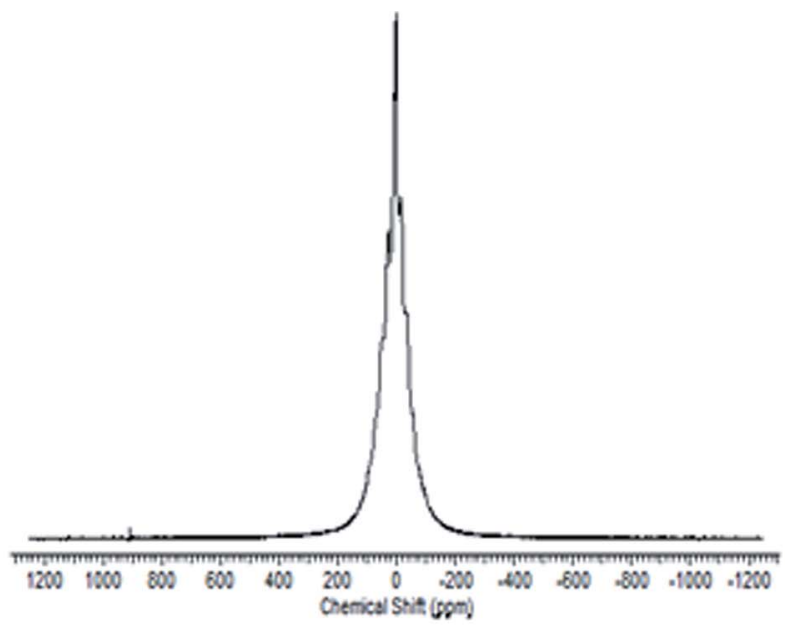

(d)

Fig. 3 Spectra of (a) DSC, (b) FTIR, (c) ${ }^{13} \mathrm{C}$, and (d) ${ }^{1} \mathrm{H}$ NMR of the compound obtained using DMF as a solvent. 
Hence, studies were carried out on prepared saturated solution of FOX-7 in DMF at $25{ }^{\circ} \mathrm{C}$. Liquid phase nitration was carried out in the DMF solutions, and further processes were carried out as abovementioned. Upon preliminary analysis, the white solids were found to be other than those of TNAA. Elemental analysis indicated the presence of $\mathrm{N}: 31.73$, C: $26.88, \mathrm{H}: 4.69$, and O: $36.7 \%$ which showed an increase in carbon and hydrogen percentages relative to that of TNAA.

Thermal analysis of the compound obtained in the DMF medium was carried out using a DSC7 and SDTA Q600. From the DSC thermogram (Fig. 3(a)), it was observed that the compound melted in the range from 264 to $280{ }^{\circ} \mathrm{C}$. Moreover, the TGA profile of this compound (Fig. S4 $\dagger$ ) showed its stability until $217{ }^{\circ} \mathrm{C}$ (TGA thermogram given in the ESI $\dagger$ ). The IR spectrum of this compound in the ATR mode (Fig. 3(b)) showed transmittance at 3314, 2991, 1712, 1635, 1485, 1354, 1102, 702, and $670 \mathrm{~cm}^{-1}$, which indicated the presence of $-\mathrm{OH}$, double bond, and carbonyl in the compound. From the IR values, it seems that the $\mathrm{OH}$ and carbonyl are adjacent to each other in a keto-enol transition. The ${ }^{13} \mathrm{C}$ NMR spectra (Fig. 3(c)) analysis showed three peaks at $50\left(\mathrm{CH}_{3} / \mathrm{CH}_{2}\right), 150(\mathrm{C}-\mathrm{OH})$, and $270(\mathrm{C}$ quat.) ppm, whereas in the solid state ${ }^{1} \mathrm{H}$ NMR spectra, a single broad peak was obtained at about 12 ppm (Fig. 3(d)). These analyses could not reveal an exact structure of the compound. However, further analysis was discontinued as the compound was found to be inferior to TNAA in view of the large carbon and low oxygen content. To minimize the carbon content in the new compound, formamide was used instead of DMF. However, the reaction resulted in the formation of ammonium nitrate, which was confirmed by elemental, thermal, and spectral analysis. Reaction of FOX-7 was also attempted in a suspension mode using DCM and carbon tetrachloride as TNAA compatible solvents. Moderate reaction of nitric acid in DCM was noticed while isolating the precipitate. In a carbon tetrachloride medium, oily material was noticed at the interface and separation was found to be difficult. Overall, liquid phase nitration did not occurred in the desired manner. The summary of liquid-liquid/suspension reactions is given in Table 1.

\subsection{Kinetics evaluation of the TNAA process}

The reactor is the most important entity of a chemical plant. Reaction kinetics in the form of mathematical models facilitates

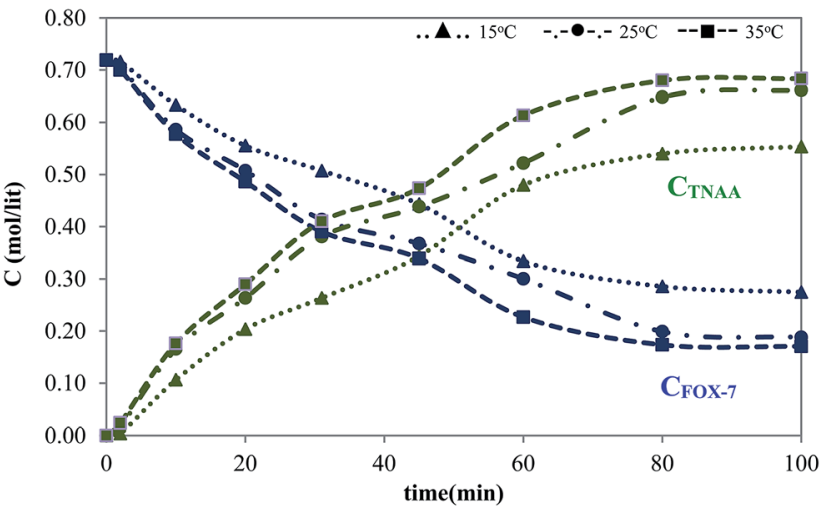

Fig. $4 \quad C-t$ profile of the TNAA reaction.

Table 2 Kinetics parameter

\begin{tabular}{llll}
\hline $\mathrm{SN}$ & $\begin{array}{l}\text { Temperature } \\
\left({ }^{\circ} \mathrm{C}\right)\end{array}$ & $\begin{array}{l}\text { Rate constant } \\
\left(\mathrm{L} \mathrm{mol}^{-1} \mathrm{~min}^{-1}\right)\end{array}$ & $\begin{array}{l}\text { Activation energy } \\
\left(\mathrm{kcal} \mathrm{mol}^{-1}\right)\end{array}$ \\
\hline 1 & 15 & 0.0203 & 5.10 \\
2 & 25 & 0.0273 & \\
3 & 35 & 0.0363 &
\end{tabular}

the design or modification of the chemical reactors and is helpful in the optimization of the product yield, effective separation of products, and elimination of environmentally harmful by-products. ${ }^{12}$ In the present study, the concentration time profile of FOX-7 and TNAA was monitored with respect to time via stopped flow analogy. ${ }^{13}$ Experiments were isothermally conducted at 15, 25, and $35{ }^{\circ} \mathrm{C}$ and for prolonged time, ensuring an insignificant change in concentrations. The observed concentration-time profile for FOX-7 and TNAA at various temperatures is given in Fig. 4 .

Fig. 4 shows that the decay pattern of FOX-7 concentration is analogous for all the temperatures. The considerable decay in the FOX-7 concentration ( 20 to $30 \%$ ) was noticed in 20 minutes of reaction time. While insignificant change in the concentration of TNAA was observed after 60 minutes of reaction time. Chemical kinetics were determined from this information and are reported in Table 2 .

Table 1 Summary of the liquid-liquid/suspension reactions

\begin{tabular}{|c|c|c|c|}
\hline SN & Solvent & Observations & Remarks \\
\hline 1 & DMF & $\begin{array}{l}\text { - FOX-7 precipitated while addition } \\
\text { of } \mathrm{HNO}_{3} \text { and dissolved on complete } \\
\text { addition of } \mathrm{HNO}_{3} \\
\text { - White fumes and brownish } \\
\text { solution observed }\end{array}$ & $\begin{array}{l}\text { - White solid having } 260^{\circ} \mathrm{C} \text { melting } \\
\text { point } \\
\text { - Analysis confirmed compound } \\
\text { other than TNAA }\end{array}$ \\
\hline 2 & Formamide & $\begin{array}{l}\text { - Violent reaction noticed with } \\
\text { white fumes }\end{array}$ & $\begin{array}{l}\text { - Reaction resulted in formation of } \\
\text { ammonium nitrate }\end{array}$ \\
\hline 4 & $\mathrm{CCl}_{4}$ & $\begin{array}{l}\text { - Two layers observed with oily } \\
\text { material at interface }\end{array}$ & $\begin{array}{l}\text { - Difficult to separate due to small } \\
\text { variation in densities }\end{array}$ \\
\hline
\end{tabular}


Kinetic evaluation of the TNAA process indicated a secondorder reaction with the FOX-7 concentration alone. The effect of nitric acid concentration on the rate was not found to be prominent due to its availability in large excess. It was also obtained that the reaction pace almost doubled at $35{ }^{\circ} \mathrm{C}$ as compared to that at $15{ }^{\circ} \mathrm{C}$. This directly helped in halving the reaction time without affecting the product quality. The activation energy of the reaction was found to be $5.1 \mathrm{kcal} \mathrm{mol}^{-1}$,

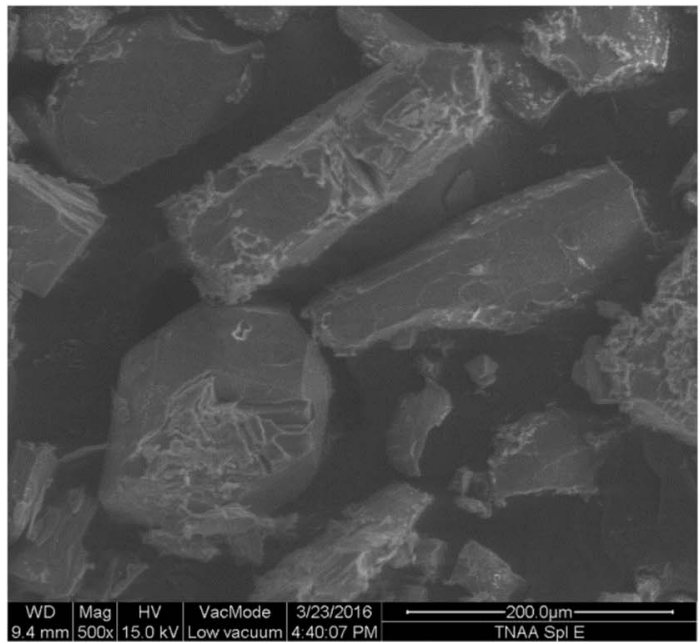

(a)

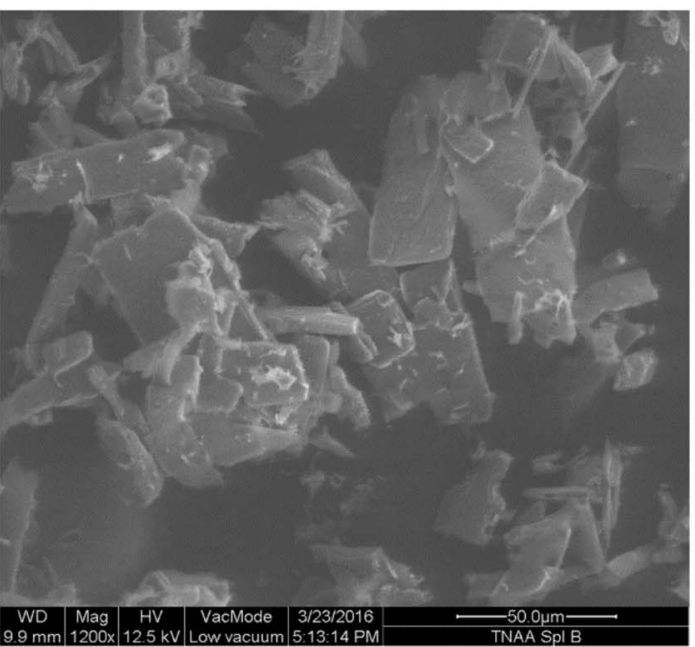

(c)

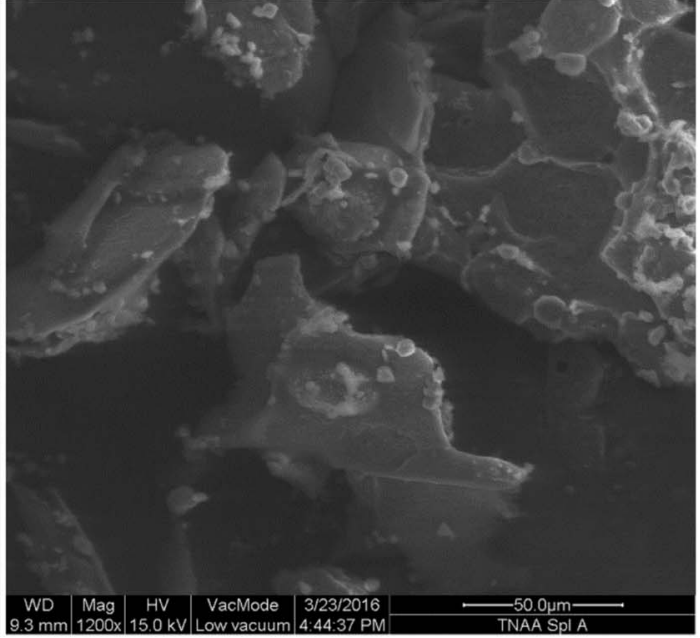

(b)

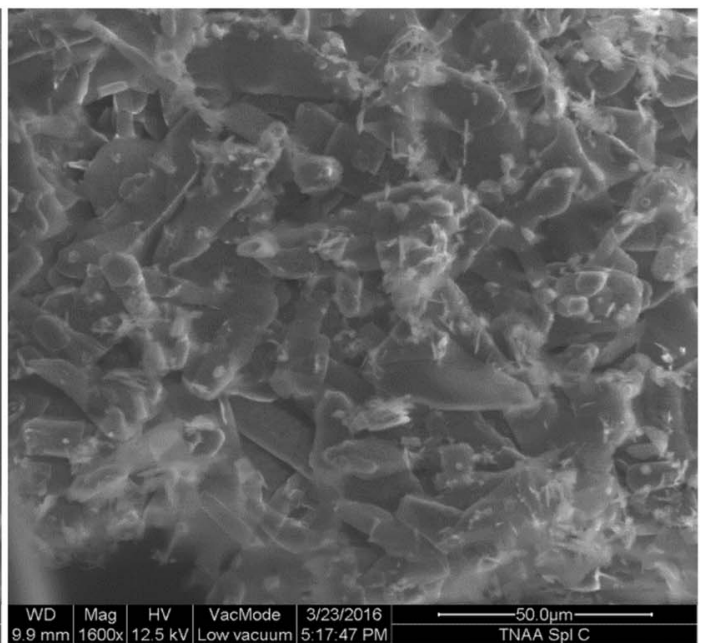

(d)

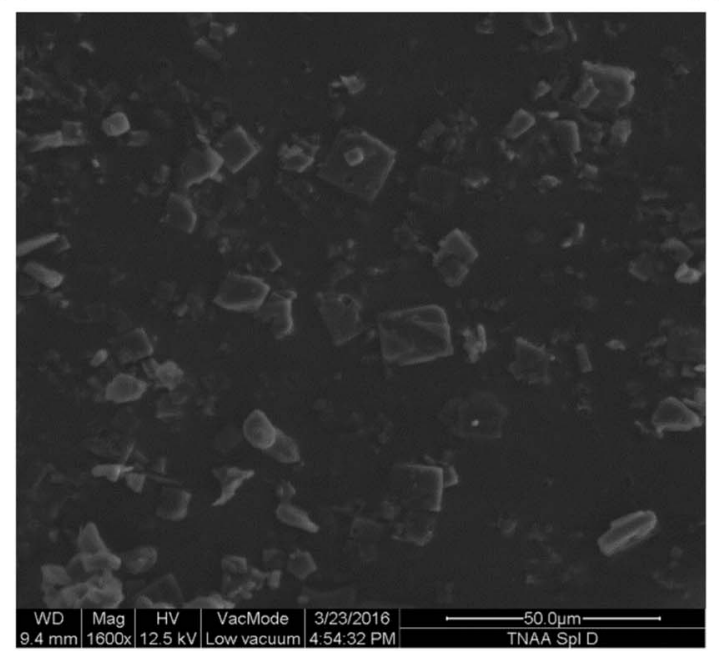

(e)

Fig. 5 SEM images of TNAA (a) crystalline and precipitated from (b) pentane, (c) hexane, (d) cyclohexane, and (e) heptane. 
Table 3 Precipitation data

\begin{tabular}{llllll}
\hline SN & Solvent & $\begin{array}{l}\text { Yield } \\
(\%)\end{array}$ & $\begin{array}{l}\text { Particle size } \\
(\mu \mathrm{m})\end{array}$ & $\begin{array}{l}\text { Surface area } \\
\left(\mathrm{m}^{2} \mathrm{~g}^{-1}\right)\end{array}$ & $\begin{array}{l}\text { Friction } \\
\text { sensitivity }(\mathrm{N})\end{array}$ \\
\hline 1. & Pentane & 90 & 2.07 & 1.55 & 118 \\
2. & Hexane & 95 & 2.14 & 1.50 & 196 \\
3. & Cyclohexane & 85 & 2.97 & 1.08 & 157 \\
4. & Heptane & 75 & 2.03 & 1.58 & 137
\end{tabular}

which also indicated the necessity of a higher reaction temperature for obtaining a higher yield in a reasonable reaction time.

\subsection{Precipitation studies}

Friction sensitivity of the ingredients of propellants is considered as one of the key characteristics for carrying out safe formulation. ${ }^{\mathbf{1 4}}$ Crystalline TNAA from a DCM medium was found to be insensitive to friction until $98 \mathrm{~N}$, which was comparable to that of $\mathrm{ADN}^{7}$ but lower than that of $\mathrm{AP}^{15}$. SEM analysis of crystalline TNAA (Fig. 5(a)) showed irregular-shaped crystals having sharp edges, which might be attributed to the friction sensitivity at $98 \mathrm{~N}$. The average particle size of crystalline TNAA was found to be $112.4 \mu \mathrm{m}$. Particle shape size management studies were carried out for improving the particle shape and thereafter friction insensitivity. A concentrated solution of TNAA in DCM $\left(130 \mathrm{~g} \mathrm{~L}^{-1}\right)$ was precipitated in 4 volume parts of pentane, hexane, cyclohexane, and heptane under constant stirring. Particle size and friction insensitivity data of these samples are provided in Table 3.

Particle size and surface area of TNAA were found to be equivalent in all the media. In the friction test, it was observed that the smaller the particles, the lower the explosive probability. ${ }^{16}$ The shape of the TNAA samples that were precipitated from pentane was found to be irregular (Fig. 5(b)) and analogous to that of the crystalline TNAA. Friction insensitivity of this sample was improved by $20 \%$ due to the smaller size of the particles $(2.07 \mu \mathrm{m})$. The maximum friction insensitivity was obtained with the TNAA sample precipitated in hexane medium and found to be 196 N. SEM analysis of this sample (Fig. 5(C)) showed very thin and uniform-sized flake-like particles. Particles of similar nature are more active for dissipation of energy in a uniform manner. The fine particle size and thin flakes of TNAA precipitated from hexane offered a dual advantage for energy dissipation by which friction insensitivity significantly improved.

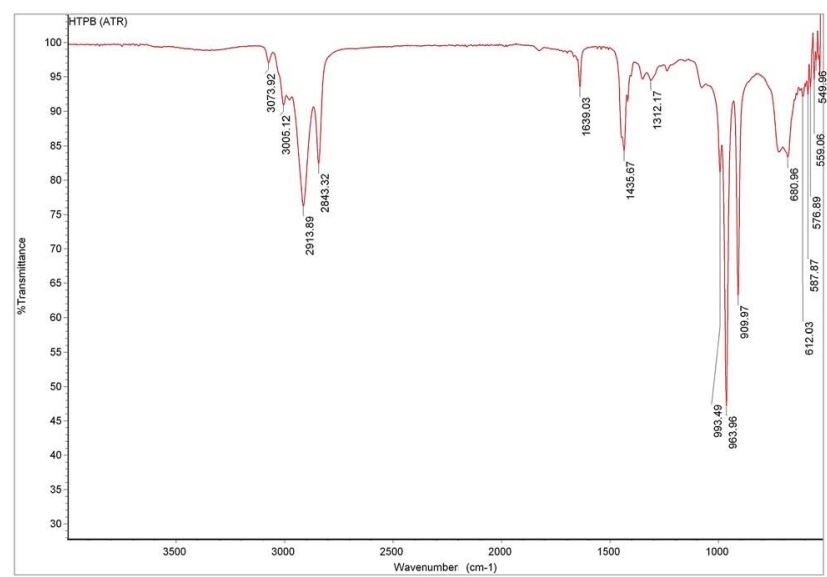

(a)

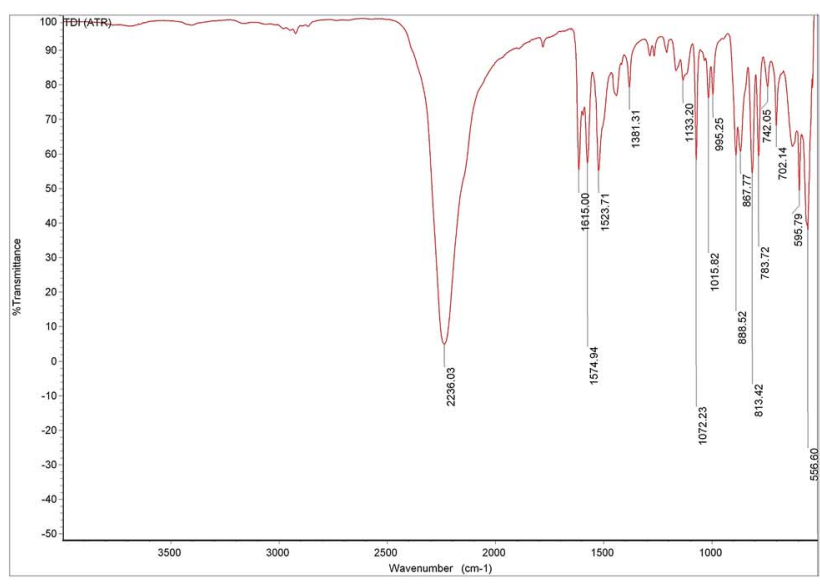

(b)

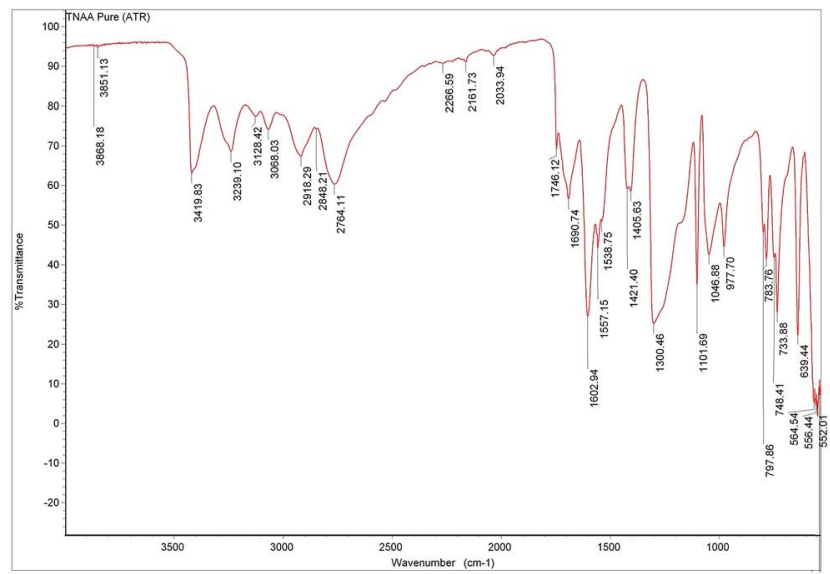

(c)

Fig. 6 FTIR spectrums of (a) HTPB, (b) TDI, and (c) TNAA. 
TNAA precipitated from cyclohexane was obtained in the shape of flakes with irregular edges (Fig. 5(d)). Hence, improvement in the friction insensitivity was obtained (157 N) and was less than that of the sample precipitated from hexane.
Block type surface with considerable thickness of TNAA was noticed in the heptane medium (Fig. 5(e)). Friction insensitivity of TNAA precipitated from heptane was found to be improved by $40 \%$ due to the regular and smaller particle size. On the other

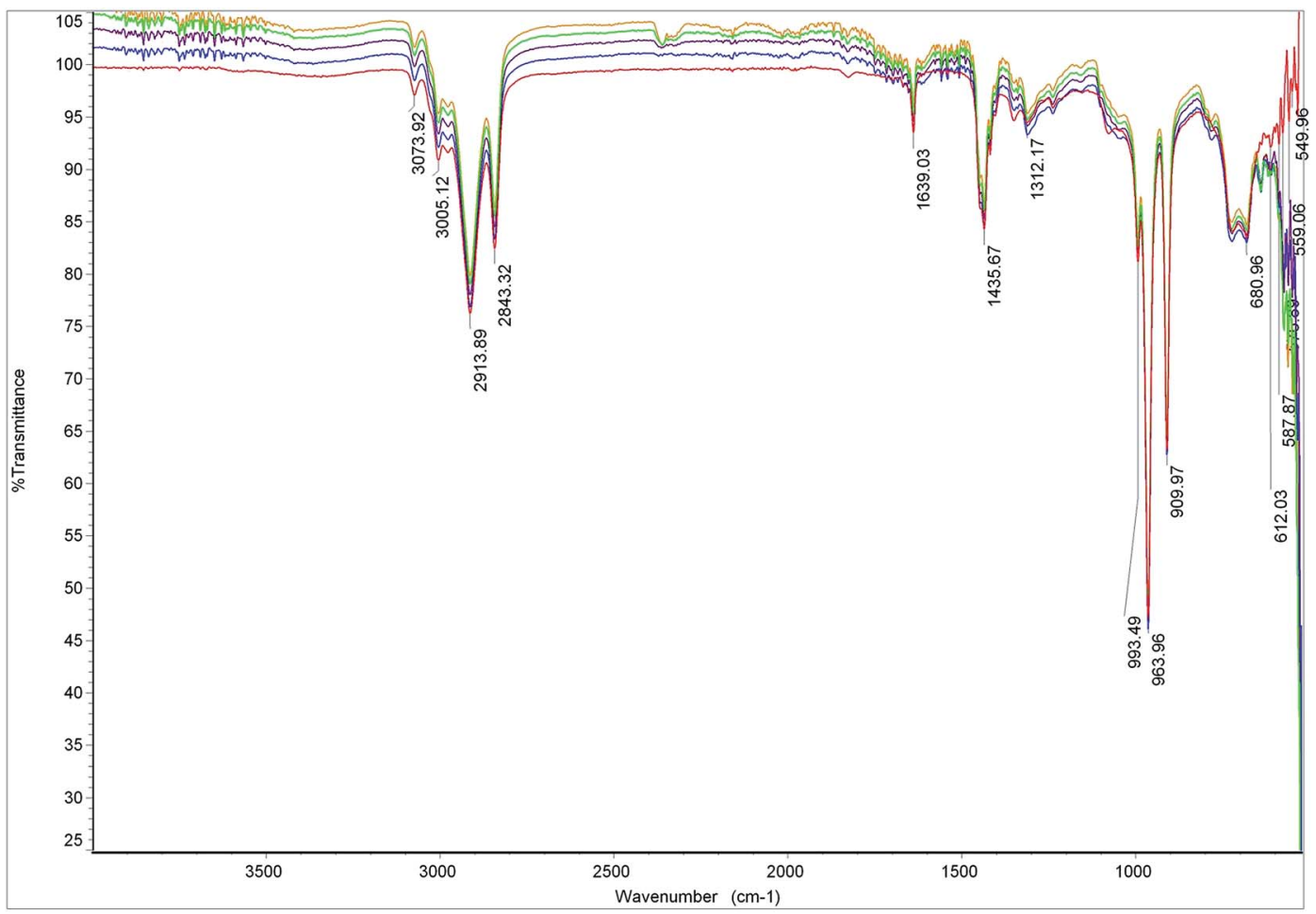

(a)

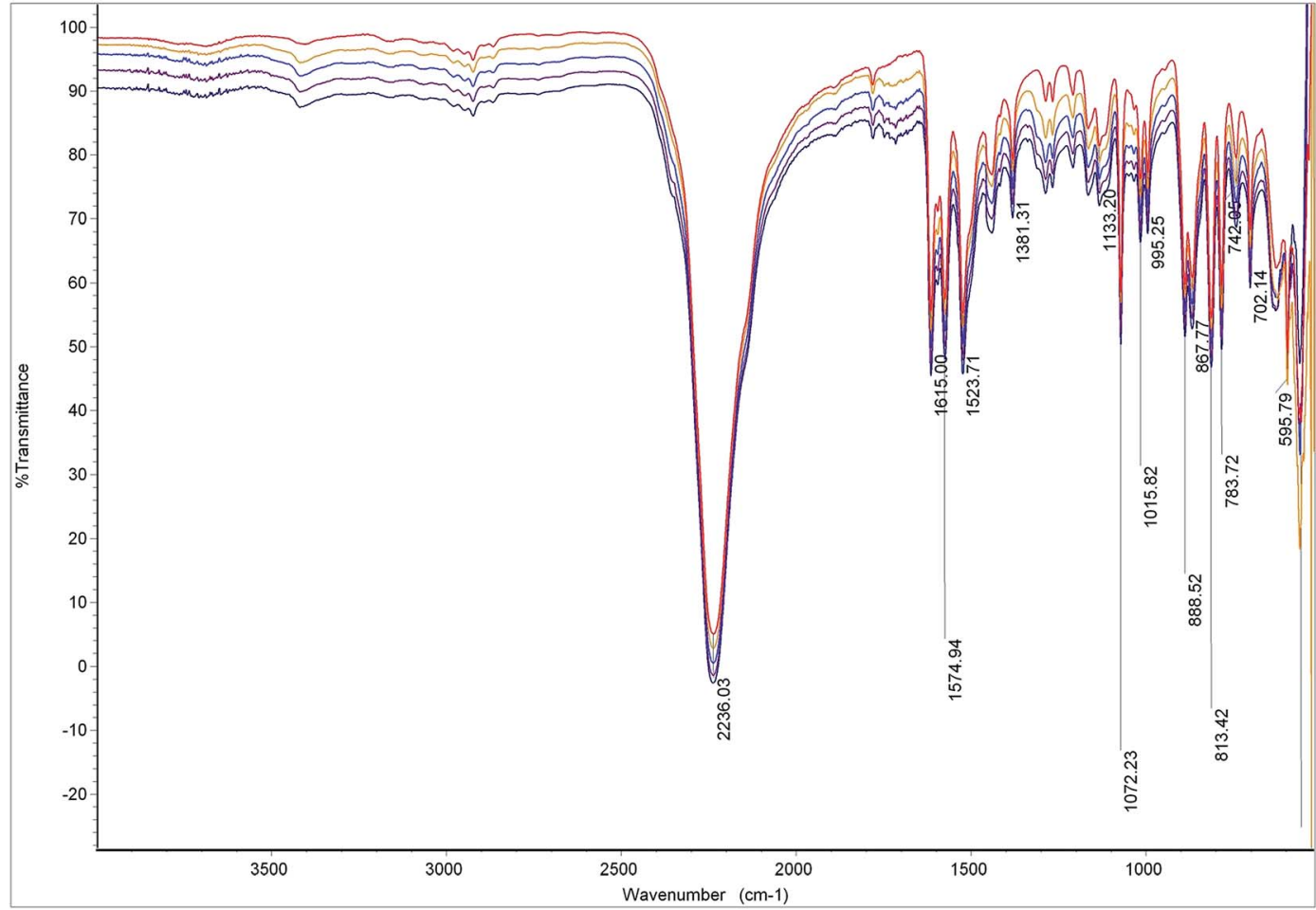

(b)

Fig. 7 FTIR spectrums of TNAA with (a) HTPB and (b) TDI. 
hand, the precipitation yield was also found to be higher (95\%) in the hexane medium, whereas it was lower in the heptane medium $(75 \%)$.

\subsection{Compatibility studies with propellant ingredients}

The application of TNAA as an oxidizer in composite propellant formulations is dependent on its compatibility with propellant ingredients, mainly toluene diisocyanate (TDI) and hydroxylterminated polybutadiene (HTPB). This is because of the known reaction between hydroxyl groups of HTPB and isocyanate groups of TDI to form polyurethane linkages, which results in the formation of a solid matrix that holds other ingredients such as oxidizer, fuel, and other additives. ${ }^{17}$ Hence, inertness of other ingredients of CP is expected with hydroxyl and isocyanate groups in view of achievement of better mechanical and aging characteristics. Herein, TNAA also exhibits hydroxyl and $\mathrm{C}=\mathrm{N}$ groups, which attracted necessity for a compatibility analysis with HTPB and TDI. Herein, spectroscopy-based technique were used for the determination of TNAA reactivity with HTPB and TDI. Initially, FTIR spectrums were obtained for HTPB, TDI, and TNAA separately. In the HTPB spectra (Fig. 6(a)), transmittance at 1639 and $2913 \mathrm{~cm}^{-1}$ confirmed the presence of $-\mathrm{C}=\mathrm{C}-$ and $-\mathrm{OH}$ functionality, respectively. The strong transmittance at
$2236 \mathrm{~cm}^{-1}$ (Fig. 6(b)) indicated the presence of the $-\mathrm{NCO}$ functionality in TDI. Fig. 6(c) shows the presence of $-\mathrm{C}-\mathrm{NO}_{2}$ (1300 and $\left.1602 \mathrm{~cm}^{-1}\right),-\mathrm{C}=\mathrm{N}-\left(1690 \mathrm{~cm}^{-1}\right)$, and $-\mathrm{OH}(3419$ $\mathrm{cm}^{-1}$ ) functionality in TNAA. These spectra were found to be in line with the spectra of pure components.

Reactivity of HTPB with TDI under different conditions has been reported by numerous authors. ${ }^{18}$ The conversion of hydroxyl functionality to urethane has been found to be easier to monitor the course of the reaction. On the same line, present study was carried out to monitor the formation of urethane/ other compound, and the disappearance of the hydroxyl and/ or cyanate functionality. HTPB-TNAA and TDI-TNAA were prepared in the standard composition and spectra were obtained for 6 hours at 30 min intervals. FTIR spectrums for these compositions are shown in Fig. 7(a) and (b). In the HTPB-TNAA compatibility analysis (Fig. 7(a)), the peak intensity of the main functionality of HTPB (-OH@2913 $\left.\mathrm{cm}^{-1}\right)$ and TNAA $(-\mathrm{C}=$ N@1690 $\mathrm{cm}^{-1}$ ) was found to be unaltered. Similarly, -NCO functionality $\left(2236 \mathrm{~cm}^{-1}\right)$ of TDI was found to be nonreactive with TNAA (Fig. 7(b)). The new prominent peaks in the range from 1690 to $1720 \mathrm{~cm}^{-1}$ corresponding to urethane formation ${ }^{18}$ were not observed during both analyses. This clearly showed the compatibility of TNAA with HTPB as well as TDI.

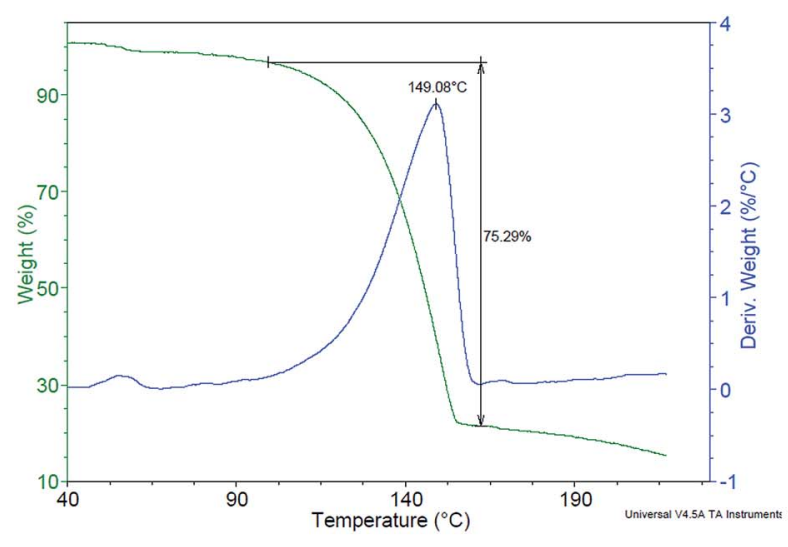

(a)

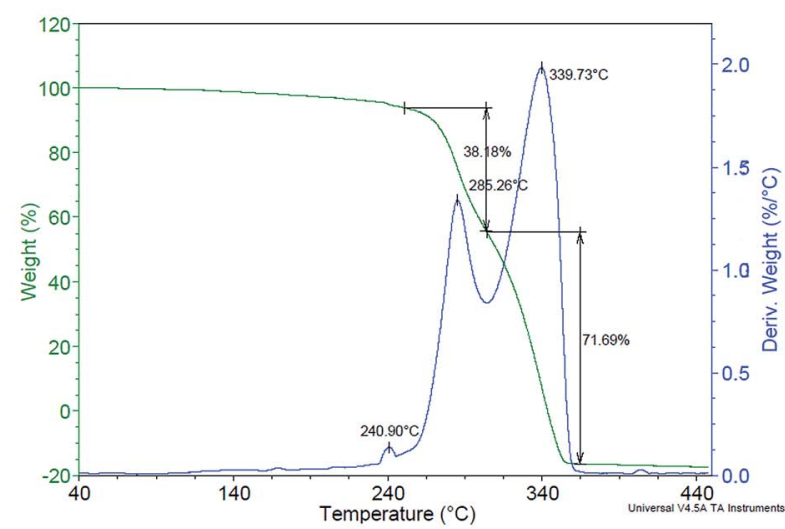

(b)

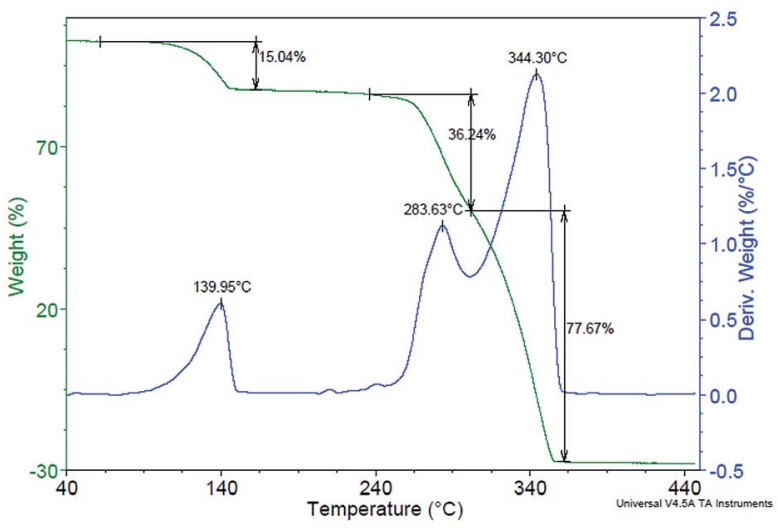

(c)

Fig. 8 TGA analysis of (a) TNAA, (b) AP, and (c) TNAA-AP mixture. 
Thermogravimetric analysis (TGA) of the TNAA-AP mixture was carried out in view of partial replacement of AP from the CP formulations. The TGA of TNAA (Fig. 8(a)) shows the onset at about $100{ }^{\circ} \mathrm{C}$ and complete decomposition at $160{ }^{\circ} \mathrm{C}$. Similarly, the TGA of AP (Fig. 8(b)) shows the onset at about $240{ }^{\circ} \mathrm{C}$ and complete decomposition at $360{ }^{\circ} \mathrm{C}$ with two phase transformation peaks at 240 and $285{ }^{\circ} \mathrm{C}$ as reported by various researchers. ${ }^{19}$ Melting of TNAA in the mixture was found to occur at about $139{ }^{\circ} \mathrm{C}$, which was lower than the melting of TNAA alone. The depression in melting may be due to a disruption in the consistency and organization of the crystal lattice at the molecular level, which weakened the lattice and enabled a more facile breakdown. On other hand, the effect of TNAA on the AP melting point was not seen in TGA. This was due to complete decomposition of TNAA before reaching the temperature essential for melting of AP. In TGA analysis, decomposition of TNAA and AP was found independently in mixture. This indicated constituents do not have effect on overall decomposition pattern, hence mentioned as indicating individuality in combustion phenomena and properties.

\section{Conclusions}

Process optimization studies are essential to obtain the operating envelope of TNAA in an efficient manner. Formation of TNAA was found to be sensitive towards the quantity of nitric acid. Appropriate quantity of nitric acid (about $35 \mathrm{~mol} \mathrm{~mol}^{-1}$ of FOX-7) is essential to obtain higher yields of TNAA and also to avoid hydrolysis. Nitric acid quantities beyond this increases the load on distillation without improving the TNAA yield. Pseudomolecular second-order kinetics was obtained due to the application of excess concentration of nitric acid. Reaction kinetics helped in identifying a temperature regime $\left(30\right.$ to $\left.35^{\circ} \mathrm{C}\right)$ by which reaction time was halved without compromising product quality and yield. During the liquid-liquid reaction, application of solvents was restricted due to compatibility issues. Usage of DMSO and NMP in the reaction resulted in decomposition of TNAA with brown fumes. On the other hand, compounds other than TNAA were obtained when DCM, DMF, and formamide were used as reaction solvents. Crystalline TNAA from DCM medium were sensitive to friction due to the formation of irregular shaped crystals with sharp edges. Friction insensitivity of TNAA significantly improved due to optimum particle size and shape. Very thin and uniform-sized flake style particles of TNAA precipitated from hexane helped in dissipating friction energy in effective manner, by which friction insensitivity significantly improved. Moreover, hexane is suitable anti-solvent in view of higher precipitation yield. Impact of particle size on friction insensitivity was obtained on comparing analogous shapes of crystalline TNAA and that precipitated from pentane. The particle size and surface area of TNAA samples precipitated from various anti-solvents are comparable, which highlights the insignificant effect of the anti-solvents. In the compatibility analysis, the functionality of HTPB and TDI in a mixture with TNAA remains unaltered. This inertness of TNAA with HTPB and TDI indicated its applicability in the composite propellant in a partial/complete manner. In view of the partial replacement of $\mathrm{AP}$ in $\mathrm{CP}$, the presence of TNAA in a mixture has not shown an effect on the decomposition of AP. Both oxidizers separately decomposed in their mixture without affecting their combustion phenomena and properties. Overall, this study provided a significant amount of information on the process intensification of insensitive TNAA and applicability of TNAA in composite propellants.

\section{Acknowledgements}

Authors are thankful to the Director HEMRL for granting permission to publish this work.

\section{References}

1 G. da Silva, S. C. Rufino and K. Iha, Green PropellantsOxidizers, J. Aerosp. Technol. Manage., 2013, 5(2), 139-144.

2 T. M. Klapötke, Chemistry of high-energy materials, Walter de Gruyter GmbH \& Co KG, 2015.

3 (a) B. $\mathrm{Gu}$ and J. D. Coates, Perchlorate: environmental occurrence, interactions and treatment, Springer Science \& Business Media, 2006; (b) W. E. Motzer, Perchlorate: problems, detection, and solutions, Environ. Forensics, 2001, 2(4), 301-311.

4 (a) E. D McLanahan, J. L. Campbell, D. C. Ferguson, B. Harmon, J. M. Hedge, K. M. Crofton, L. Braverman, D. A. Keys and M. Mumtaz, Low-dose effects of ammonium perchlorate on the hypothalamic-pituitary-thyroid axis of adult male rats pretreated with PCB126, Toxicol. Sci., 2007, 97(2), 308-317; (b) J. Dumont, The Effects of Ammonium Perchlorate on Reproduction and Development of Amphibians, DTIC Document, 2008; (c) R. E. Tarone, L. Lipworth and J. K. McLaughlin, The epidemiology of environmental perchlorate exposure and thyroid function: a comprehensive review, J. Occup. Environ. Med., 2010, 52(6), 653-660.

5 (a) R. Zajtchuk and R. Bellamy, Textbook of military medicine. Part, 1997; (b) A. Makhijani and K. Gurney, Mending the ozone hole: science, technology, and policy, Mit Press, 1995.

6 C. Oommen and S. Jain, Ammonium nitrate: a promising rocket propellant oxidizer, J. Hazard. Mater., 1999, 67(3), 253-281.

7 J. I. S. Oliveira, ADN-The new oxidizer around the corner for an environmentally friendly smokeless propellant, J. Aerosp. Technol. Manage., 2009, 1(2), 153.

8 P. Dendage, D. Sarwade, S. Asthana and H. Singh, Hydrazinium nitroformate (HNF) and HNF based propellants: a review, J. Energ. Mater., 2001, 19(1), 41-78.

9 T. T. Vo, D. A. Parrish and J. N. M. Shreeve, Tetranitroacetimidic Acid: A High Oxygen Oxidizer and Potential Replacement for Ammonium Perchlorate, J. Am. Chem. Soc., 2014, 136(34), 11934-11937.

10 R. V. Carr and R. J. Schmitt, Nitration: recent laboratory and industrial developments, Amer Chemical Society, 1996.

11 J. H. Ahn, J. K. Kim, H. S. Kim, E. J. Kim and K. K. Koo, Solubility of 1, 1-diamino-2, 2-dinitroethylene in $\mathrm{N}, \mathrm{N}$ dimethylformamide, dimethyl sulfoxide, and $N$-methyl-2pyrrolidone, J. Chem. Eng. Data, 2009, 54(12), 3259-3260. 
12 O. Levenspiel, Chemical reaction engineering, Ind. Eng. Chem. Res., 1999, 38(11), 4140-4143.

13 (a) J. F. Eccleston, J. P. Hutchinson and H. D. White, Stoppedflow techniques. Protein Ligand Interactions: Structure and Spectroscopy. A Practical Approach Series, ed. S. E. Harding and B. Z. Chowdry, 2001, pp. 201-237; (b) S. E. Harding and B. Z. Chowdhry, Protein-ligand interactions, structure and spectroscopy: a practical approach, Oxford University Press, USA, 2001.

$14 \mathrm{H}$. Bazaki and N. Kubota, Friction sensitivity mechanism of ammonium perchlorate composite propellants, Propellants, Explos., Pyrotech., 1991, 16(1), 43-47.

15 D. Fischer, T. M. Klapötke and J. Stierstorfer, Chem. Commun., 2016, 52(5), 916-918.
$16 \mathrm{X}$. Song, Dependence of particle size and size distribution on mechanical sensitivity and thermal stability of hexahydro-1, 3, 5-trinitro-1, 3, 5-triazine, Def. Sci. J., 2009, 59(1), 37.

17 C. R. Nair, J. U. Bhaskar and K. Ninan, Polyurethanes from Hydroxyl Terminated Polybutadiene: Effect of Hydroxyl Value on Crosslinking and Mechanical Properties, J. Polym. Mater., 2013, 30(4), 363.

18 M. I. Aranguren and R. J. Williams, Kinetic and statistical aspects of the formation of polyurethanes from toluene diisocyanate, Polymer, 1986, 27(3), 425-430.

19 L. Mallick, S. Kumar and A. Chowdhury, Thermal decomposition of ammonium perchlorate-A TGA-FTIRMS study: Part I, Thermochim. Acta, 2015, 610, 57-68. 